\title{
Desenvolvimento de recurso de tecnologia assistiva para paciente com lesão do plexo braquial
}

\section{Development of an assistive technology feature for a patient with brachial plexus injury}

\author{
Flauber Felipe de Oliveira Santos ${ }^{1}$, Eliza Ribeiro de Oliveira ${ }^{2}$
}

http://dx.doi.org/10.11606/issn.2238-6149.v28i2p246-253

Santos FFO, Oliveira ER. Desenvolvimento de recurso de tecnologia assistiva para paciente com lesão do plexo braquial. Rev Ter Ocup Univ São Paulo. 2017 maio-ago.;28(2):246-53.

RESUMO: Um dos desafios cotidianos para pessoas com Lesão do Plexo Braquial (LPB) é desempenhar as Atividades de Vida Diária (AVDs) com sucesso, desde atividades simples, como o vestir, até atividades mais complexas como preparar uma refeição. Tais pessoas podem ser beneficiadas pelas Tecnologias Assistivas (TAs), que são definidas como qualquer item, equipamento ou produto, seja ele adquirido comercialmente, modificado ou personalizado que é usado para aumentar, manter ou melhorar as capacidades funcionais dos indivíduos. O objetivo deste estudo é relatar a experiência de desenvolvimento de um recurso de TA para um paciente com LPB da clínica escola de Terapia Ocupacional da Universidade Federal da Paraíba (UFPB). Primeiramente foi realizada a avaliação com o uso da Medida Canadense de Desempenho Ocupacional (COPM) para identificar as necessidades do paciente. Após o desenvolvimento do produto foram realizados treinos de uso e o acompanhamento em ambiente domiciliar, com vista a testar sua eficácia. $\mathrm{O}$ paciente ainda passou por um processo de reavaliação, com a reaplicação da COPM para comparação dos resultados. De acordo com os resultados é possível considerar o recurso eficaz, por aumentar o desempenho e a satisfação do paciente quanto à realização da atividade que estava afetada.

DESCRITORES: Plexo braquial/lesões; Equipamentos de autoajuda; Adaptação; Terapia ocupacional; Atividades cotidianas.
Santos FFO, Oliveira ER. Development of an assistive technology feature for a patient with brachial plexus injury. Rev Ter Ocup Univ São Paulo. 2017 May-Aug.;28(2):246-53.

ABSTRACT: One of the daily challenges for people with Brachial Plexus Injury (BPI) is to perform Activities of Daily Living (ADLs) successfully, from simple activities such as dressing up, to more complex activities, like preparing a meal. Thereby these people can benefit from Assistive Technologies (ATs), which are defined as any item, equipment or product, whether purchased commercially, modified or customized that is used to increase, maintain or improve the functional capabilities of individuals. The purpose of this study is to report the development of an AT resource for a patient with BPI from the clinic of Occupational Therapy School of the Federal University of Paraíba. Firstly, an evaluation was performed using the Canadian Occupational Performance Measure (COPM) to identify the patient's needs. After the development of the product, user training was carried out in order to test its effectiveness. A re-evaluation process has been conducted, with the reapplication of COPM to compare the results. According to the results it is possible to consider that the resource is effective, increasing the satisfaction of the patient in performing the activity that was affected.

KEYWORDS: Brachial plexus/injury; Self-help devices; Adaptation; Occupational therapy; Activities of daily living.

Este artigo é resultado do Trabalho de Conclusão de Curso de Flauber Felipe de Oliveira Santos "Desenvolvimento de um recurso de Tecnologia Assistiva para um paciente com lesão do plexo braquial", 2016. Apresentado no XI Congresso Norte Nordeste de Terapia Ocupacional, Fortaleza, CE, 2016.

1. Terapeuta Ocupacional graduado pelo Departamento de Terapia Ocupacional, Universidade Federal da Paraíba, João Pessoa, PB, Brasil. E-mail: flauber_12@hotmail.com

2. Curso de Terapia Ocupacional do Departamento de Terapia Ocupacional da Universidade Federal da Paraíba, João Pessoa, PB, Brasil. E-mail: eliza@lavid.ufpb.br

Endereço para correspondência: Universidade Federal da Paraíba, Departamento de Terapia Ocupacional - Campus I, s/n. Castelo Branco, João Pessoa, PB, Brasil. CEP: 58051-900. 


\section{INTRODUÇÃO}

Lesão do Plexo Braquial (LPB) é uma
patologia que pode causar incapacidade e
afetar a participação social e a realização das Atividades de Vida Diárias (AVDs) dos indivíduos acometidos. Devido à perda de força, alterações de sensibilidade, subluxação de ombro e dores fortes no membro atingido, indivíduos com LPB tem dificuldade em se readaptar ao cotidiano e retomar as suas AVDs ${ }^{1}$.

Estudos mostram que $10 \%$ a $20 \%$ das lesões do sistema nervoso periférico envolvem o Plexo Braquial (PB). Dessas, $80 \%$ a $90 \%$ são causadas por traumas auto/motociclísticos ${ }^{2}$. Nos casos de danos ou sequelas funcionais, a utilização de equipamentos de Tecnologia Assistiva (TA) pode ser uma das possibilidades para resgatar a autonomia dos indivíduos atingidos.

O termo "Assistive Technology", ou Tecnologia Assistiva, surgiu pela primeira vez em 1988 na legislação norte-americana Public Law 100-407, componente do "ADA - American with Disabilities Act". De acordo com o ADA, TA é definida como qualquer item, equipamento ou sistema de produto, seja ele adquirido comercialmente, modificado ou personalizado, que é usado para aumentar, manter ou melhorar as capacidades funcionais dos indivíduos com deficiência ${ }^{3}$.

De acordo com o Comitê de Ajudas Técnicas $\mathrm{CAT}^{4}$ do Brasil, a TA é definida como:

Uma área do conhecimento, de característica interdisciplinar, que engloba produtos, recursos, metodologias, estratégias, práticas e serviços que objetivam promover a funcionalidade, relacionada à atividade e participação de pessoas com deficiência, incapacidades ou mobilidade reduzida, visando sua autonomia, independência, qualidade de vida e inclusão social (p.9).

Neste sentido, é possível afirmar que a TA tem como objetivo proporcionar à pessoa com deficiência e/ou limitações, independência, autonomia e inclusão.

De acordo com a classificação do "International Organization for Standardization" ISO 99995, a TA possui três níveis diferentes de especificação, sendo "Classe", "Subclasse" e "Detalhamento da classificação". O primeiro nível de classificação tem onze classes de produtos de TA, entre as quais está a classe "Cuidados com o Lar". Dentro desta classificação, os recursos adaptados para as AVDs relacionadas com alimentação estão inseridos na categoria dos Produtos de Apoio para preparação de comidas e bebidas, denominada como "Produtos de apoio para cortar, picar e separar alimentos". Reis $^{6}$ classifica TA de acordo com seu nível de tecnologia sendo estes, alta tecnologia - Dispositivos eletrônicos e computadores; média tecnologia Dispositivos que incorporam elementos de mecânica com grau intermediário de complexidade; baixa tecnologia Itens de pouca sofisticação, como instrumentos adaptados para alimentação, e nenhuma tecnologia - Soluções que se restringem a procedimentos e serviços. Esta última não utiliza dispositivos ou equipamentos.

A baixa tecnologia abrange recursos adaptados e confeccionados com objetos pouco sofisticados que auxiliam nas atividades de vida diária e que não requerem, necessariamente, gastos com materiais. As adaptações facilitam o desempenho nas atividades que estão diretamente ligadas às ocupações humanas, como as atividades de se alimentar, cozinhar, vestir-se, tomar banho, entre outras ${ }^{7}$.

$\mathrm{Na}$ Terapia Ocupacional, o termo adaptação refere-se a modificações no ambiente, na tarefa ou no método, que objetivam a maximização da funcionalidade do indivíduo e maior grau de independência possível no desempenho da atividade significativa para o paciente ${ }^{8}$. Para Kielhofner', a Terapia Ocupacional é uma área da saúde que atua na construção ou reconstrução de hábitos e/ou ocupações humanas, voltadas para as atividades que não são mais realizadas, mas que o indivíduo gostaria de realizá-las. Dessa forma, o uso de TA pela Terapia Ocupacional pode trazer benefícios com relação ao desempenho ocupacional, com o objetivo de promover independência e autonomia na realização de certas atividades.

Para Alves et al. ${ }^{10}$, o terapeuta ocupacional é um dos profissionais responsáveis pela prescrição e implementação de recursos de TA na realidade brasileira. Em uma pesquisa realizada por esses autores, foi possível identificar em quais modalidades os recursos de tecnologia são confeccionados por Terapeutas Ocupacionais. Em ordem crescente está em primeiro lugar produtos para Atividades de Vida Diária e as Atividades Instrumentais de Vida Diária, seguida de Adequação Postural, juntamente com Computadores e Adaptação Ambiental. Logo após encontra-se a Comunicação Alternativa, Órteses, Adaptações Escolar, Prótese e, por fim, Adaptações para Carro. De acordo com o Conselho Federal de Fisioterapia e Terapia Ocupacional (COFFITO) $)^{11}$, compete ao terapeuta 
ocupacional prescrever, orientar, executar e desenvolver produtos, recursos, metodologias, estratégias, práticas e serviços de TA como elementos constituintes ao processo de intervenção terapêutico ocupacional.

Além disso, cabe ao T.O realizar a avaliação cinesiológica afim de identificar as capacidades remanescentes do paciente, possibilitando propor o recurso mais adequado. A avaliação dos Membros Superiores (MMSS) tem o objetivo de identificar a Amplitude de Movimento (ADM) e movimentos inerentes ao MMSS. Tais movimentos podem incluir extensão, flexão, adução, abdução, rotação interna e externa do ombro; extensão e flexão do cotovelo; pronação e supinação do antebraço; extensão e flexão, desvio radial e ulnar do punho e a extensão e flexão, adução e abdução das falanges. De acordo com Cavalcanti e Galvão ${ }^{12}$ :

Para o terapeuta ocupacional, a principal preocupação é verificar se a ADM articular disponível possibilita o desempenho de atividades de cuidados pessoais, atividades produtivas e vocacionais, de lazer e sociais. Portanto, o terapeuta avalia a amplitude de movimento tendo em vista os seguintes objetivos: examinar a amplitude de movimente disponível; determinar as limitações que afetam a função; identificar as limitações que podem produzir deformidade; avaliar a necessidade de uso órtese, aparelhos de assistência ou ambos; estabelecer os objetivos de tratamento apropriados; selecionar as modalidades de tratamento adequadas, como técnicas de posicionamento e outras estratégias para diminuir as limitações; manter um registro da progressão ou regressão do quadro (p.75).

No caso da LPB, alguns dos movimentos de MMSS citados acima não são realizados devido ao comprometimento dos nervos, o que contribui para a dificuldade em realizar diversas atividades.

Neste sentido, o presente estudo tem o objetivo de relatar, através de um estudo de caso, a experiência de desenvolvimento de um recurso de TA para um paciente com LPB da clínica escola de Terapia Ocupacional da Universidade Federal da Paraíba (UFPB). Além disso, objetiva-se verificar a eficácia do produto através de treinos de uso e da avaliação da satisfação do paciente com relação ao recurso.

Foi realizada uma avaliação inicial que inclui a elaboração do perfil ocupacional, avaliação cinesiológica e a aplicação da Medida Canadense de Desempenho Ocupacional (COPM), a qual possibilitou especificar as necessidades do paciente. Foi elaborado, então, um produto de TA voltado para suas necessidades. Após o desenvolvimento do produto, foram realizados treinos de uso com o paciente e o acompanhamento em ambiente domiciliar, a fim de testar sua eficácia. Ainda foi feito um processo de reavaliação que incluiu reaplicar a COPM para comparar os resultados e detectar possíveis ganhos e benefícios após a utilização da adaptação.

\section{METODOLOGIA}

A pesquisa realizada fundamenta-se em um estudo de caso de sujeito único com LPB, no qual o paciente apresentou-se disposto e interessado em fazer parte. De acordo com Ventura ${ }^{13}$, um estudo de caso ocorre quando se analisa, de forma detalhada, um caso individual, permitindo explicar a dinâmica e a patologia de uma doença específica. O estudo de caso como estratégia de pesquisa também pode ser entendido como uma metodologia. A fim de manter o sigilo, o paciente será denominado "E". O projeto foi aprovado pelo Comitê de Ética sob $\mathrm{n}^{\circ} \mathrm{CAAE}$ 55983416.2.0000.5188 e o paciente assinou o Termo de Consentimento Livre e Esclarecido (TCLE).

Foram realizados cinco encontros com duração média de duas horas cada na clínica escola de Terapia Ocupacional da UFPB. No primeiro encontro foi realizada uma avaliação inicial do paciente, a qual inclui a elaboração de um perfil ocupacional, a partir de uma entrevista sobre sua história de vida. Durante este processo, também foi realizada uma avaliação cinesiológica, afim de detectar movimentos remanescentes no membro acometido. No segundo encontro a COPM foi aplicada com o propósito de identificar as demandas funcionais do paciente, bem como detectar mudanças com relação a percepção sobre seu desempenho ocupacional e mudanças em sua satisfação com relação a esse desempenho. Um diário de observação foi realizado pelo terapeuta ocupacional com o propósito de documentar todo o processo de intervenção. $\mathrm{O}$ estudo foi fundamentado na metodologia de Reis ${ }^{6}$, que determina a produção de um produto TA em oito etapas (Gráfico 1).

Por fim, após o desenvolvimento do produto, foram realizados dois encontros na clínica, nos quais ocorreram treinos com o paciente, a fim de testar a eficácia do produto. O paciente ainda passou por um processo de reavaliação (quinto e último encontro na clínica escola) com a reaplicação da COPM para comparar os resultados, possíveis ganhos e os benefícios após a utilização do recurso. 
Gráfico 1 - Etapas para confecção de um recurso de TA de Reis

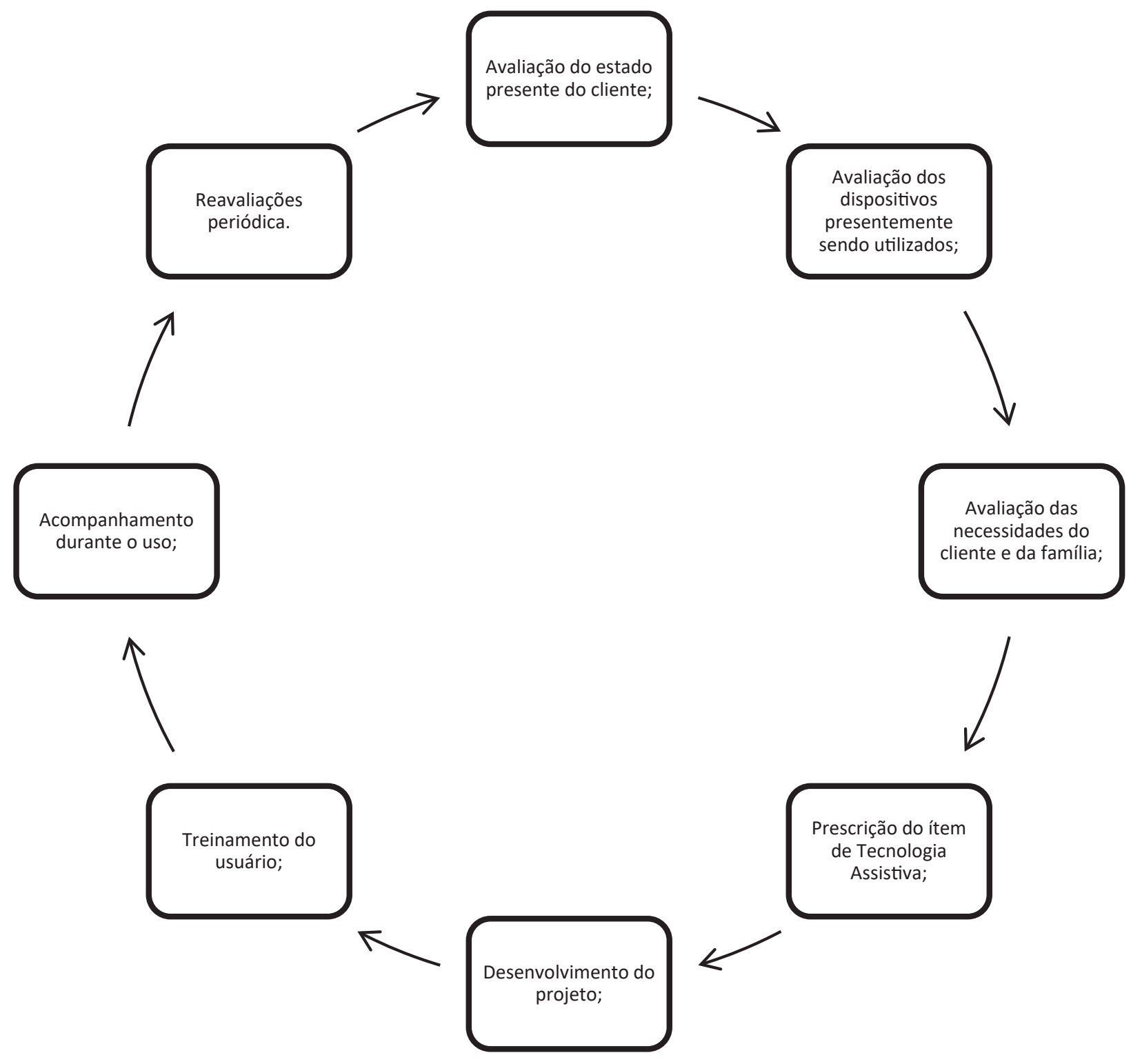

\section{RESULTADOS}

Abaixo encontram-se apresentadas as etapas do processo de elaboração do recurso de TA.

\section{1) Perfil ocupacional do paciente e avaliação cinesiológica}

"E", 33 anos, sexo masculino, relata que sofreu um acidente motociclístico no ano de 2008 que resultou em um traumatismo cranioencefálico e avulsão do PB. Passou por várias cirurgias, inclusive a de enxerto e realocação dos nervos que compõem o PB. Devido à gravidade do acidente, "E" perdeu a função no membro superior direito. Portanto, qualquer movimento que realiza é de forma passiva, com a ajuda do membro superior esquerdo, não apresentando ADM ativa no membro acometido. O membro dominante de "E" é o direito, o que aumenta a dificuldade na realização de suas atividades. A utilização de tipoia é necessária para evitar que o membro fique edemaciado. 
Devido ao quadro atual o paciente sente dificuldade em realizar atividades bimanuais, o que impede a realização de AVDs importantes como preparar uma refeição, alimentar-se sozinho e atividades de autocuidado. "E" costuma reunir familiares e amigos em casa para fazer churrasco e queixa-se de que gostaria de cortar a carne e servir, ao contrário de ser servido. Relata que o churrasco é uma atividade de lazer significativa. Mora com sua família, a qual inclui sua esposa, dois enteados e a mãe. Além disso, tem uma filha do seu primeiro casamento que reside com a avó. Atualmente trabalha em um escritório de contabilidade e nas horas vagas trabalha como técnico de manutenção de dispositivos tecnológicos, como aparelhos celulares e Notebooks.

\section{2) Definição de metas}

De acordo com o perfil do paciente, viu-se a necessidade de aplicar a COPM, já que o mesmo mostra dificuldade em realizar várias tarefas em seu cotidiano. A Tabela 1 apresenta os resultados da COPM. Dentre os problemas, surgiram a dificuldade para escrever, cortar e descascar alimentos, abotoar a camisa e para amarrar o cadarço. Como demonstrado na Tabela 1, o problema "Amarrar o cadarço" apresenta "1" em desempenho e satisfação, assim como o resultado apresentado no problema "Cortar e descascar alimentos". "E" relatou que todos os seus calçados fechados eram de elástico, ficando então como prioridade o "Cortar e descascar alimentos".

Tabela 1 - Resultado da avaliação inicial da COPM

\begin{tabular}{llccc}
\hline Área & Problemas Encontrados & Importância & Desempenho & Satisfação \\
\hline Produtividade & 1 - Escrever & 10 & 7 & 7 \\
& 2 - Cortar e descascar alimentos & 10 & 1 & 1 \\
\hline Autocuidado & 1 - Abotoar a camisa & 10 & 6 & 6 \\
& 2 - Amarrar o cadaç̧o & 10 & 1 & 1 \\
\hline
\end{tabular}

\section{3) Confecção do produto de TA}

A partir do exposto acima, optou-se pela confecção de uma tábua adaptada para facilitar a fixação de alimentos, objetivando ampliação da independência no manuseio e no corte dos alimentos. A idealização da tábua também considerou a atividade de lazer como fator relevante, já que o churrasco com amigos e familiares é significativo para o paciente. Uma visita domiciliar foi feita no intuito de analisar possíveis barreiras arquitetônicas e compreender as demandas locais do domicílio para a confecção do recurso. Foram observados fatores como a altura da mesa, altura da pia e o posicionamento dos móveis. É importante ressaltar que dentre as oito etapas para elaboração de um recurso de TA, não foi utilizado a etapa "Avaliação dos dispositivos presentemente sendo utilizados", uma vez que o paciente não utilizava nenhum recurso anterior ao estudo.

Para elaborar a tábua foram utilizados os seguintes materiais: tábua em bambu $25 \times 35$, com pega em silicone; 8 parafusos tamanho 6 galvanizados; 4 parafusos tamanho 7 galvanizados; 6 ventosas em silicone para fixação sobre diferentes superfícies. Foram escolhidos parafusos do tipo inoxidáveis, que diminuem o risco de sofrer corrosão.

Foram realizados treinos de uso da tábua na clínica escola e em ambiente domiciliar. Para o treino na clínica foram levados vários alimentos presentes nas refeições cotidianas, como: bife de carne assada, peito de frango, pão, batata inglesa (cozida e crua). A tábua foi posicionada em frente ao paciente sobre uma mesa. $\mathrm{O}$ mesmo permaneceu sentado e os alimentos foram expostos e usados de forma aleatória. O principal objetivo da tábua é fixar os alimentos enquanto o paciente manuseia os alimentos. Todos os movimentos foram realizados com o membro esquerdo. A Figura 1 corresponde ao momento do treino na clínica, descascando uma batata inglesa cozida.

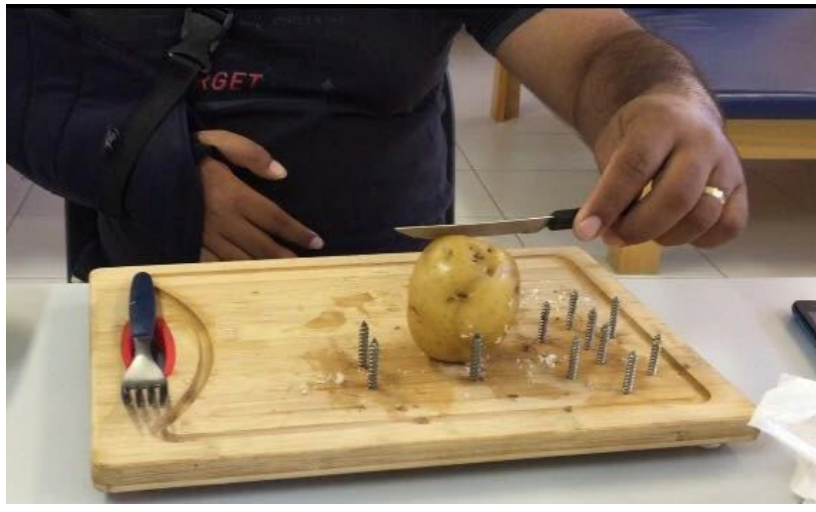

Figura 1 - Treino de uso na clínica escola de Terapia Ocupacional da UFPB 
"E" não apresentou nenhuma dificuldade nos treinos de uso da tábua na clínica escola, relatando ser um recurso funcional que será muito útil para o seu dia a dia e que está em busca da sua melhora e independência. Foi possível observar o entusiasmo ao realizar a atividade de corte e o descascar, bem como a facilidade em que o mesmo teve ao manusear a faca sobre a tábua.

Os treinos de uso no ambiente domiciliar foram realizados sem orientações para o paciente, de forma a observar o manuseio livre da tábua. Foi notório que, por estar em casa, "E" sentiu-se mais a vontade. Foram realizados treinos na cozinha, local de preparo das refeições, e na área de serviço da casa, local destinado aos momentos de lazer, neste caso, o churrasco. Foi possível observar todo o preparo para o churrasco com o uso da tábua durante o momento de lazer, no qual o paciente demonstrou estar satisfeito, chegando a mostrar o recurso para os amigos presentes. Não foi apresentada nenhuma dificuldade em utilizar a tábua. "E" optou que a tábua não fosse fixada em nenhum local específico, devido a facilidade e praticidade de ser levada para qualquer ambiente domiciliar.

\section{Resultado final da COPM}

A COPM foi reaplicada após dois meses de uso do recurso elaborado com o intuito de investigar as mudanças com relação ao desempenho e nível de satisfação do paciente na tarefa "cortar um alimento". Este resultado pode trazer informações no que diz respeito à eficácia do produto confeccionado. Além disso, os resultados trazem informações sobre a independência e autonomia com relação à atividade e se o paciente usou ou não a adaptação. A tabela a seguir (Tabela 2) corresponde aos resultados finais relacionados à alimentação.

A partir dos resultados da Tabela 2, é possível identificar a diferença existente entre "Desempenho e Satisfação 1" e "Desempenho e Satisfação 2", resultados estes obtidos através da reaplicação do instrumento. Este resultado corrobora com a fala do paciente, que diz estar satisfeito com o recurso por proporcionar a realização de uma atividade que estava impossibilitado de fazer, mudando a situação de dependente para independente.

"E" relata que usa o recurso com mais frequência nos finais de semana e nos dias que está em casa, principalmente pela manhã ao preparar o café da manhã. Afirmou que tem sucesso em cerca de $90 \%$ dos alimentos que consome usando a tábua e que procura sempre utilizar o recurso. O paciente afirma que irá continuar treinando suas habilidades na tábua para conseguir fazer com maior rapidez as tarefas que envolvem seu uso.

Tabela 2 - Resultado da reavaliação da COPM

\begin{tabular}{llccccc}
\hline Área & Problemas Encontrados & Importância & Desempenho 1 & Satisfação 1 & Desempenho 2 & Satisfação 2 \\
\hline Produtividade & 1 - Cortar e descascar alimentos & 10 & 1 & 1 & 9 & 10 \\
\hline
\end{tabular}

\section{DISCUSSÃO}

Os resultados, tanto relacionados à COPM quanto as observações realizadas pelo terapeuta, apontam que o produto atendeu às necessidades e expectativas do paciente com relação à tarefa de cortar alimentos. Isso indica que existe baixa possibilidade de desuso ou abandono do recurso produzido, uma vez que o mesmo foi elaborado a partir de demandas e necessidades específicas do paciente. Phillips e Zhao ${ }^{14}$ afirmam que a falta de consideração pela opinião dos usuários de um produto ocasiona baixo desempenho nas tarefas, mesmo após a utilização da tecnologia, e que mudanças nas necessidades e prioridades dos usuários, são as principais causas de abandono de um produto de TA.

Outro fator que atua no sentido de evitar o desuso é a eficiência do produto. De acordo com Veytizou et al. ${ }^{15}$, um produto é eficaz quando possibilita aos utilizadores obter o resultado esperado. Já um produto eficiente permite alcançar o resultado pretendido com menor esforço. Ainda de acordo com Veytizou et al. ${ }^{15}$, a eficácia está ligada diretamente com o resultado final do produto, sendo determinado apenas pelo fato de conseguir ou não atingir o objetivo. Neste aspecto, pode-se afirmar que o produto confeccionado foi eficaz e que maior eficiência pode ser atingida com maior número de treinos. Outras melhorias poderiam ser realizadas no sentido de aumentar a eficiência do produto.

Além disso, Veytizou et al. ${ }^{16}$, afirma que, quando o design é centrado no usuário, possibilita uma análise das habilidades motoras do paciente, viabilizando o processo de verificação das suas necessidades, sua deficiência e a definição das especificidades físicas no geral, possibilitando a elaboração de um produto que se adeque ao 
máximo às demandas identificadas. De acordo com Albanez et al. ${ }^{17}$, os pacientes cuja expectativa em relação aos produtos não é atendida serão insatisfeitos, enquanto os pacientes cuja expectativa foi alcançada ou até mesmo superada são considerados como satisfeitos. Tendo em vista as falas de "E" e os resultados da COPM, é possível afirmar que o mesmo ficou satisfeito com o produto e com os resultados finais adquiridos. É possível afirmar que "E" atingiu seu objetivo a partir do momento em que conseguiu cortar, não somente a batata, mas também a carne do churrasco durante o momento de lazer. Seguindo essa proposta, o recurso foi elaborado especificamente para as necessidades do paciente e, dessa forma, há maiores chances de sucesso na utilização do produto.

Outro ponto a ser destacado é a facilidade de acesso aos materiais para confecção do recurso elaborado, o que torna o mesmo viável por possuir um preço acessível e por ser elaborado a partir de produtos de fácil aquisição. Apesar de existirem poucos estudos na Terapia Ocupacional que comprovam eficácia dos produtos de baixo custo, sabe-se que o terapeuta ocupacional é um profissional apto para trabalhar e desenvolver TAs com o intuito de promover a independência, autonomia e inclusão social dos individuos? ${ }^{7}$. A associação dos conhecimentos do profissional de TO relacionados às TAs, juntamente com a utilização de instrumentos padronizado e validados específicos da profissão, dá maior credibilidade ao estudo e permite maior respaldo científico ao estudo desenvolvido. Além disso, a comprovação da satisfação do paciente e melhor desempenho na tarefa desejada a partir da utilização de um instrumento,

\section{REFERÊNCIAS}

1. Meneses KVP, Rocha DN, Júnior FLC, Sono T, Oliveira AL, Pinotti M. Aplicação de uma órtese funcional para mão em indivíduos com lesão do plexo braquial. Rev Ter Ocup Univ São Paulo. 2012;23(1):34-43. doi: http://dx.doi. org/10.11606/issn.2238-6149.v23i1p34-43.

2. Mumenthaler M. Some clinical aspects of peripheral nerve lesions. Eur Neurol. 1969;2(5):257-68. doi: $10.1159 / 000113802$.

3. Brasil. Secretaria Especial dos Direitos Humanos (SEDH). Tecnologia assistiva. Brasília; 2009 [citado 2009]. Disponível em: http://www.pessoacomdeficiencia.gov.br/ app/publicacoes/tecnologia-assistiva.

4. Brasil. Secretaria Nacional de Promoção dos Direitos da Pessoa com Deficiência (SNPD). Secretaria de Direitos cuja credibilidade baseia-se em "teste e reteste", confirma o potencial da ação do terapeuta ocupacional na elaboração de produtos de TA ${ }^{18,19}$. Neste sentido, de acordo com o resultado inicial e a reaplicação da COPM, é possível observar a mudança significativa que ocorreu através do uso do recurso e verificar que a intervenção foi eficaz, comprovando mais uma vez a confiabilidade que a COPM apresenta.

\section{CONSIDERAÇÕES FINAIS}

As Tecnologias Assistivas têm conquistado cada vez mais espaço na área da saúde, principalmente na Terapia Ocupacional, pois tem o intuito de proporcionar autonomia e independência, aumentando ou devolvendo a função afetada de quem faz uso das mesmas. Quando este recurso é desenvolvido centrado nas necessidades do paciente, há maiores chances de sucesso na utilização destes. No presente caso, "E" conseguiu atingir seu objetivo a partir da utilização do recurso de TA confeccionado, comprovando a funcionalidade do mesmo. Além da funcionalidade, a utilização do recurso trouxe a satisfação de realizar uma atividade que estava afetada.

Neste sentido, este estudo pode ser visto como uma contribuição para literatura sobre TAs de baixo custo, ao apresentar materiais de fácil acesso e preço não elevado, além disso, é importante ressaltar que o envolvimento do terapeuta ocupacional é relevante, através do engajamento e conhecimento sobre o desenvolvimento destes recursos, por estarem relacionados a todas as áreas de ocupação no intuito de melhorar o desempenho ocupacional.
Humanos da Presidência da República. Secretaria Especial dos Direitos da Pessoa com Deficiência. Brasília; 2012 [citado 2012]. Disponível em: http://www. pessoacomdeficiencia.gov.br/app.

5. Brasil. ISO 9999:2007 - Anexo III - Lista homologada [citado 28 abr. 2016]. Disponível em: http://www.inr.pt/uploads/ ISO $\% 209999 \% 201$ ista $\% 20 a \% 20$ publicar1_convertido.pdf.

6. Reis NMN. Ações inclusivas de sucesso. In: Anais III Seminário Internacional Sociedade Inclusiva PUC Minas. Belo Horizonte; 2004. p.1-5.

7. Bersch R. Introdução a tecnologia assistiva. In: Assistiva, tecnologia e educação. Porto Alegre, RS [citado em 2013] Disponivel em: http://www.assistiva.com.br/Introducao_ Tecnologia_Assistiva.pdf. 
8. Araujo PMP. Adaptações. In: Greve JMA. Tratado de medicina de reabilitação. São Paulo: Roca; 2007. p.325-9.

9. Kielhofner G. Self-reports: eliciting client's perspectives. In: Model of human occupation: theory and application. 4th ed. Baltimore: Lippincott Williams \& Wilkins; 2008. p.237-61. doi: http://dx.doi.org/10.1080/01642120802647733.

10. Alves ACJ, Emmel MLG, Matsukura TS. Formação e prática do terapeuta. Rev Ter Ocup Univ São Paulo. 2012;23(1):24-33. doi: http://dx.doi.org/10.11606/issn.22386149.v23i1p24-33.

11. COFITO - Resolução $n^{\circ} 458$, de 20 de novembro de 2015 - Dispõe sobre o uso da tecnologia assistiva pelo terapeuta ocupacional e dá outras providências. [citado 20 nov. 2015] Disponível em: http://www.coffito.gov.br/nsite/?p=3221.

12. Rodrigues AMVN, Alves GBO. Métodos e técnicas de avaliação em componentes de desempenho. In: Cavalcanti e Galvão. Fundamentos da terapia ocupacional. Rio de Janeiro: Koogan; 2007. p.75.

13. Ventura MM. O estudo de caso como modalidade de pesquisa. Rev SOCERJ. 2007;20(5):383-6.

14. Phillips B, Zhao H. Predictors of assistive technology abandonment. J Assistive Technol. 1993;5(3):36-45. http:// dx.doi.org/10.1080/10400435.1993.10132205
15. Veytizou J, Thomann G, Villeneuve F. Un produit universel pour une interface sur mesure. In: Colloque Jeunes Chercheurs et Jeunes Chercheuses. Paris, France; 2013. p.1.

16. Veytizou J, Magnier C, Villeneuve F, Thomann G. Integrating the human factors characterization of disabled users in a design method. Application to an interface for playing acoustic music. Assoc Adv Modelling Simulation Techn Enterprises. 2012;73(3):173.

17. Albanez JAPA, Garcia SFAG, Galli LCLA. Métodos de pesquisa e satisfação de clientes: um estudo bibliométrico. Rev Bras Pesq Marketing Opinião Mídia. 2014;(16):1-27. Disponível em: https://gaussconsulting.com.br/blog/metodos-de-pesquisa-desatisfacao-de-clientes-um-estudo-bibliometrico/.

18. Hohmann P, Cassapian MR. Adaptações de baixo custo. Rev Ter Ocup Univ São Paulo. 2011:22(1):10-18. doi: http:// dx.doi.org/10.11606/issn.2238-6149.v22i1p10-18.

19. Caldas ASC, Facundes VLD, Silva HJ. O uso da Medida Canadense de Desempenho Ocupacional em estudos brasileiros: uma revisão sistemática. Rev Ter Ocup Univ São Paulo 2011;22(3):238-44. doi: http://dx.doi.org/10.11606/ issn.2238-6149.v22i3p238-244

Recebido em: 01.12.2016

Aceito em: 28.07.2017 